Research Article

\title{
Non-invasive Blood Glucose Measurement based on Impedance Spectrum
}

\author{
Tong Yin, Xiaoyan Chen*, Meng Du, Hongyi Yin \\ College of Electronic Information and Automation, Tianjin University of Science and Technology, China
}

\section{ARTICLE INFO}

Article History

Received 25 October 2018

Accepted 19 November 2018

Keywords

Blood glucose concentration impedance spectroscopy non-invasive glucose measurement

\begin{abstract}
Different from the traditional measurement approach and instruments of blood glucose concentration, we propose a novel measuring method related to electrical impedance spectrum without invasion, and we have researched the correspondence between blood glucose values and impedance parameters. Considering the skin contacting impedance and cytomembrane conductivity feature, an equivalent circuit of human model was built in the MATLAB Simulink environment. We obtained the impedance parameters of the model at $1 \mathrm{~Hz}-20 \mathrm{MHz}$ by simulation. The measured data were analyzed by a Cole-Cole model to derive the relationship between the impedance value and the blood glucose concentration. The results show that the electrical impedance is strong negative correlation to blood glucose concentration, as the blood glucose concentration increases, the impedance value decreases. The conclusion of this research provides a theoretical basis for non-invasive blood glucose surface-measuring.
\end{abstract}

(C) 2020 The Authors. Published by Atlantis Press SARL.

This is an open access article distributed under the CC BY-NC 4.0 license (http://creativecommons.org/licenses/by-nc/4.0/).

\section{INTRODUCTION}

The blood glucose concentration is one of the key indicators reflecting the condition of diabetic patients. At present, the most common testing method is acupuncture measurement before and after meal invasively, which bring great pain to the patients with potential dangers of infection [1]. Compared with the common invasive method, non-invasive blood glucose concentration measuring has the advantages of no acupuncture and no pains with simple and quick operation. Non-invasive blood glucose concentration measuring has an important application value of monitoring the blood glucose change in time and of visualizing the abnormally glucose metabolism rapidly [2].

This aim of this research is to explore a new way that detecting the blood glucose concentration non-invasively by electrical impedance scanning (EIS) method on the surface of body. The concentration of electrolytes in the blood and tissues changes with glucose concentration, which in turn causes an imbalance in electrolyte concentration between blood and tissue fluid. This causes the ions to move in a directional manner, and the electrical properties of the cytomembrane change with the concentration of ions passing through the cytomembrane. From a macro perspective, the impedance of the human body will also change accordingly. On the biological principle, it indicates a hopeful direction for non-invasive blood glucose measuring.

Corresponding author.Email: cxywxr@tust.edu.cn

\section{THE RELATIONSHIP DEDUCTION OF GLUCOSE AND CYTOMEMBRANE CAPACITANCE}

An increasing concentration of glucose in blood induces significant reactions in blood and human tissues. It is known that the concentration of sodium decreases and potassium increases due to the active transport of ions and water movement from the tissue and blood cells, such as erythrocytes, into the vascular system. It can be understood on the cellular level that glucose uptake through membrane transporters requires controlling the electrolyte balance to maintain osmolality and cell sizes [3].

Shap et al. [4] proposed and verified the relationship between cytomembrane capacitance $\left(C_{\mathrm{mem}}\right)$ and blood glucose concentration $\left(C_{\mathrm{glu}}\right)$ by Michaelis-Menten equation as

$$
\frac{C_{\text {mem }}}{C_{\text {mem }, 0}}=1+\frac{0.53}{1+\frac{8.57}{C_{\text {glu }}}}
$$

According to the experimental data in Beving et al. [5], the cytomembrane capacitance value is $1 \mu \mathrm{F} / \mathrm{cm}^{2}$ when the blood glucose concentration is zero. In this paper, a rectangular electrode of $3 \mathrm{~cm}^{2}$ is selected for simulation test, so $C_{\text {mem, } 0}=3 \mu \mathrm{F}$. We bring $C_{\text {mem, }}$ into the Equation (1), and obtain the relationship between the cytomembrane capacitance values corresponding to the blood glucose levels of different concentrations, as shown in Equation (2), and as shown in Figure 1. 


$$
C_{\mathrm{mem}}=3+\frac{1.59}{1+\frac{8.57}{C_{\mathrm{glu}}}}
$$

From Figure 1, at the change stage of 3-10 $\mathrm{mmol} / \mathrm{L}$ in human blood glucose concentration, the cytomembrane capacitance changes very rapidly, and this change is more obvious than that caused by other substances to the cytomembrane capacitance. When the cytomembrane capacitance changes, the influence of other substances can be ignored. Therefore, the impedance index can be used to indicate the blood glucose reasonably.

\section{EQUIVALENT CIRCUIT MODEL}

To simulate the effects of different blood glucose concentrations on impedance spectroscopy measurements, we established a human equivalent circuit model. In an alternating electric field, the biological tissue has greater impedance at the lower frequency. The capacitive impedance characteristic of biological tissues was discovered by scholar Philippson in 1920, who established a corresponding bio-impedance equivalent circuit model.

Due to the detecting electrodes and skin contacting, it is also necessary to consider the effect of contact impedance. Therefore, we established a novel human equivalent circuit model as shown in Figure 2.

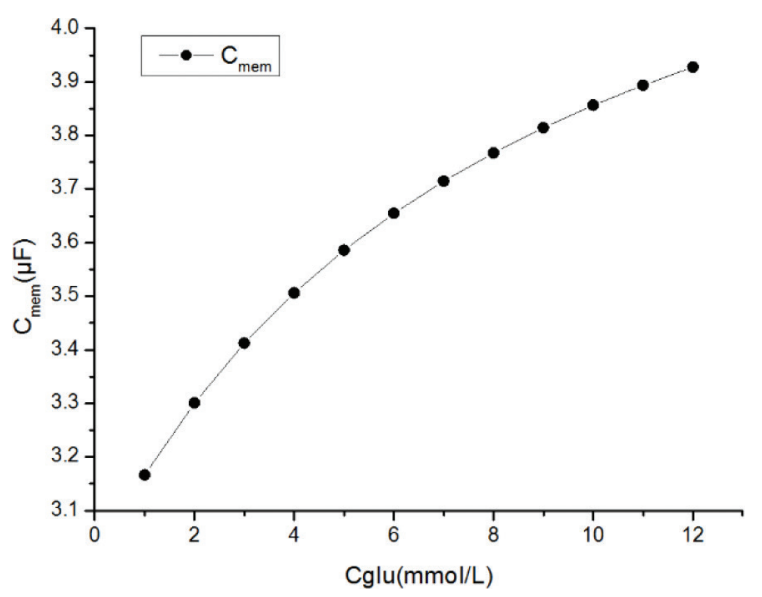

Figure $1 \mid$ The relationship between $C_{\mathrm{glu}}$ and $C_{\mathrm{mem}}$. As the $C_{\mathrm{glu}}$ increases, $C_{\text {mem }}$ shows an upward trend.

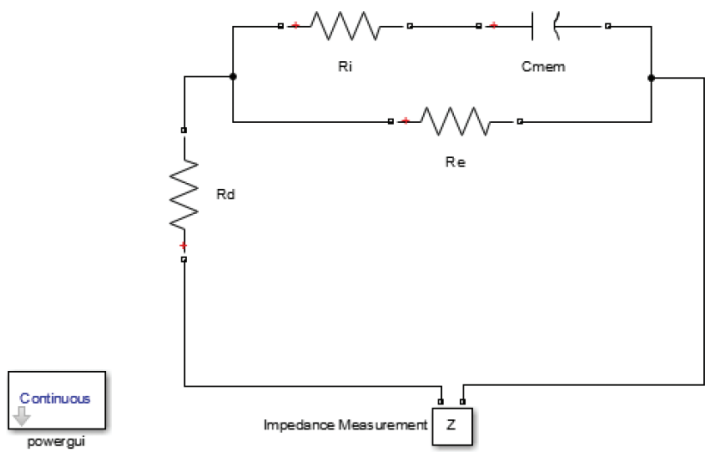

Figure 2 Human equivalent circuit model.
$R_{d}$ represents the contact impedance, $R_{i}$ is the internal liquid resistance of the human body, $R_{e}$ is the liquid resistance, and $C_{\text {mem }}$ is the cytomembrane capacitance. According to the experimental data in Chen et al. [6], we set $R_{d}=1 \mathrm{k} \Omega, R_{i}=500 \Omega$, $R_{e}=500 \Omega$.

\section{DATA PROCESSING AND RESULT ANALYSIS}

In order to accurate describe the equivalent circuit model of biological tissues, Cole and Cole $[7,8]$ have conducted in-depth research in this regard. They summed up a set of actual biological tissue electrical impedance theory, the Cole-Cole theory, and used it to describe the dielectric model of biological tissue. The basic content of the Cole-Cole theory is that the trajectory of the electrical tissue's electrical impedance in the complex plane is a circular arc in the fourth quadrant, and the center of the circle is not on the real axis but in the first quadrant.

The measurement of bio-impedance is essentially to obtain the characteristic parameters in the impedance trajectory map. It can be seen from the above analysis that as long as an excitation electric signal of a certain frequency is applied to the biological tissue and the output signal flowing through the biological tissue is simultaneously measured, the impedance amplitude at the frequency can be calculated by the relevant demodulation algorithm. If the excitation power signal is operated in a sweep mode, the entire impedance circle can be obtained by curve fitting. On the impedance plot, the impedance value at any frequency point can be obtained. Based on this theory, the Cole-Cole chart and its electrical characteristic parameters of the human equivalent circuit model are obtained from the real and imaginary parts of the impedance and the phase and impedance values. Corresponding Cole-Cole biological tissue electrical impedance characteristic equation is:

$$
\mathrm{Z}=R_{\infty}+\frac{R_{0}-R_{\infty}}{1+j w \frac{1}{2 \pi f}}
$$

The $I_{m}$ axis represents the imaginary part of the impedance, and the $R_{e}$ axis represents the real part value. $f_{c}$ represents the frequency value when the imaginary part is maximum. $R_{0}$ represents the electrical impedance of biological tissue at a frequency of zero. $R_{\infty}$ represents the electrical impedance of biological tissue at infinite frequency. These scatter points in the figure are calculated at frequencies from $10 \mathrm{~Hz}$ to $20 \mathrm{MHz}$, in which the impedance changes from 1500 to $1250 \Omega$. According to the measured real and imaginary parts of the impedance, the Cole-Cole circle is fitted through Python using the weighted average method, and the following Figure 3 shows the Cole-Cole circle fitted at $C_{\text {glu }}=6 \mathrm{mmol} / \mathrm{L}$. Fitted into a circle by these scatter plots and it can be seen that it is normative.

The change trend of the characteristic parameter $f_{c}$ has a great correlation with the change of the blood glucose concentration. From the data in Table 1 , the magnitude of $f_{c}$ has an inverse correlation with the magnitude of the blood glucose concentration. The higher blood glucose concentration, the smaller value of $f_{c}$ corresponding to the impedance spectrum. 


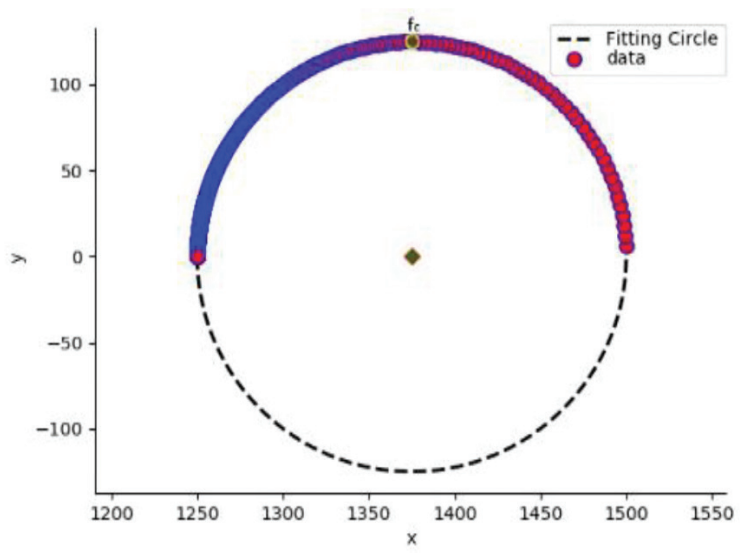

Figure 3 The Cole-Cole impedance circle.

Table 1 Impedance spectrum characteristic parameters and corresponding blood glucose concentration

\begin{tabular}{lcc}
\hline Diagnosis & $\boldsymbol{f}_{\boldsymbol{c}}(\mathbf{H z})$ & $\boldsymbol{C}_{\mathrm{glu}}(\mathbf{m m o l} / \mathrm{L})$ \\
\hline Hypoglycemia & 48.22 & 2 \\
Hypoglycemia & 45.40 & 4 \\
Normal glucose & 43.55 & 6 \\
Abnormal glucose metabolism & 42.24 & 8 \\
Abnormal glucose metabolism & 41.27 & 10 \\
Diabetes & 40.52 & 12 \\
\hline
\end{tabular}

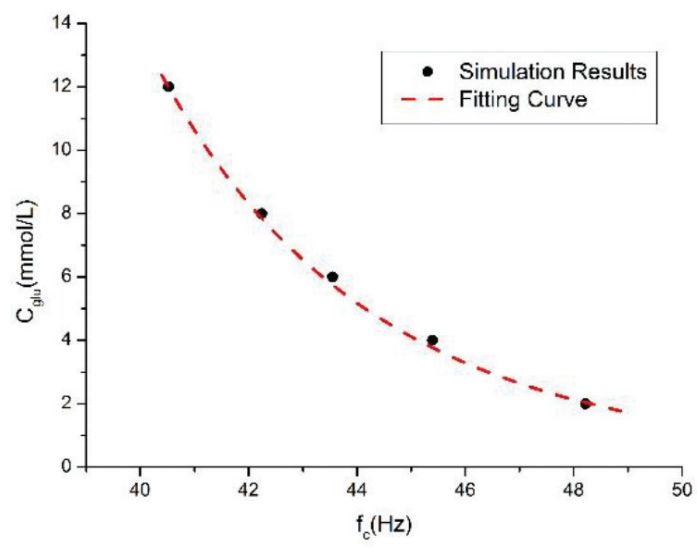

Figure $4 \mid$ Power function fitting.

A power function fitting was performed by MATLAB to obtain a correspondence between $C_{\mathrm{glu}}$ and $f_{c}$ :

$$
C_{\mathrm{glu}}=3 \times 10^{17} f_{c}^{-10.2}
$$

Power function fitting results are shown in Figure 4.

The coefficient of determination $R^{2}=0.9981$, which means that the fitting effect is excellent. The characteristic frequency $f_{c}$ in the impedance spectrum can reflect the change of human blood glucose concentration to a certain extent, and it is possible to realize non-invasive blood glucose monitoring based on impedance spectrum method.

\section{CONCLUSION}

As the glucose concentration changes, the binding of glucose to the transporter changes the capacitance of the cytomembrane. The transport of glucose into cells by transporters is a macroscopic change in the impedance of the human body. Considering the skin contacting impedance and cytomembrane conductivity feature, an equivalent circuit of human model was built in the MATLAB Simulink environment. We obtained the impedance parameters of the model at $1 \mathrm{~Hz}-20 \mathrm{MHz}$ by simulation. The measured data were analyzed by a Cole-Cole model and the blood glucose concentration, and a power function fitting was performed by MATLAB to obtain a correspondence between $C_{\text {glu }}$ and $f_{c}$ as shown in Equation (4). The results show that the electrical impedance is strong negative correlation to blood glucose concentration, especially the characteristic frequency $f_{c}$, as the blood glucose concentration increases, the impedance value decreases. The conclusion of this research provides a theoretical basis for non-invasive blood glucose surface-measuring.

\section{CONFLICTS OF INTEREST}

The authors declare they have no conflicts interest.

\section{ACKNOWLEDGMENTS}

This paper was supported by a grant from the National Natural Science Foundation of China $(61301246,41704131)$ and Tianjin Science and Technology Key Project (18YFZCGX00360).

\section{REFERENCES}

[1] A.G.A. Aggidis, J.D. Newman, G.A. Aggidis, Investigating pipeline and state of the art blood glucose biosensors to formulate next steps, Biosens. Bioelectron. 74 (2015), 243-262.

[2] D.C. Klonoff, Noninvasive blood glucose monitoring, Diabetes Care 20 (1997), 433-437.

[3] A. Caduff, E. Hirt, Y. Feldman, Z. Ali, L. Heinemann, First human experiments with a novel non-invasive, non-optical continuous glucose monitoring system, Biosens. Bioelectron. 19 (2003), 209-217.

[4] P. Shao, X. Zhang, C. Zouxue, Biological tissue impedance model for blood glucose monitoring, J. Laser Biol. 25 (2016), 250-256.

[5] H. Beving, L.E.G. Eriksson, C.L. Davey, D.B. Kell, Dielectric properties of human blood and erythrocytes at radio frequencies (0.2-10 MHz); dependence on cell volume fraction and medium composition, Eur. Biophys. J. 23 (1994), 207-215.

[6] Y. Chen, Y. Cao, Y. Gao, S.H. Pun, P.U. Mak, M.I. Vai, et al., Experimental study on contact impedance of intra-body communication transmitter, J. Biomed. Eng. Res. 29 (2010), 13-17.

[7] K.S. Cole, R.H. Cole, Dispersion and absorption in dielectrics I, Alternating current characteristics, J. Chem. Phys. 9 (1941), 341-351.

[8] K.S. Cole, R.H. Cole, Dispersion and absorption in dielectrics II. Direct current characteristics, J. Chem. Phys. 10 (1942), 98-105. 


\section{AUTHORS INTRODUCTION}

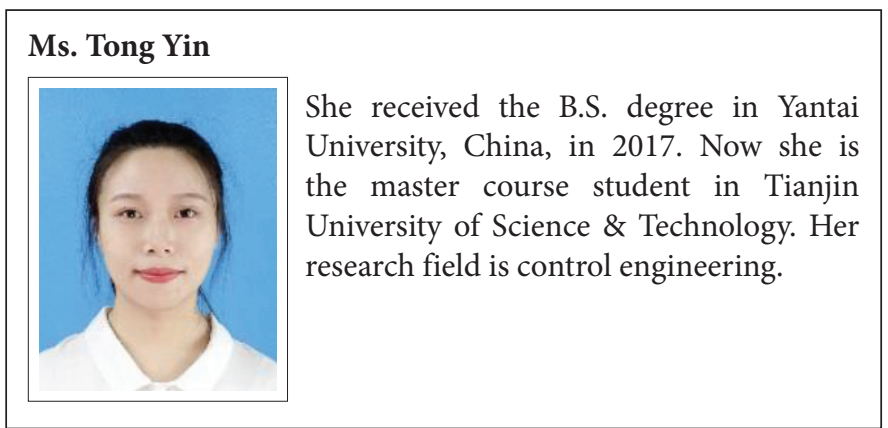

\section{Dr. Xiaoyan Chen}

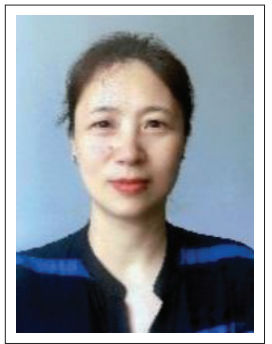

She received the M.S. degree from the Tianjin University of Science and Technology, in 1999, and the $\mathrm{PhD}$ degree in measurement technology and automation devices from Tianjin University, in 2009. From 2009 to 2015, she held a post-doctoral position at Tianjin University. She was invited by RPI, USA, as a Visiting Scholar, from 2009 to 2010, and Kent, U.K., in 2012. She is currently a Professor and an Advisor of Postgraduate and Doctorate students with the Tianjin University of Science and Technology.

\section{Dr. Meng Du}

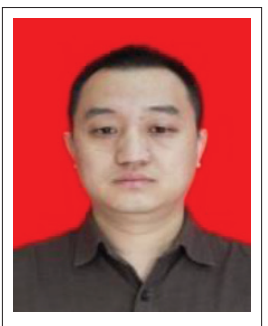

He received the B.S. degree in computer science and technology from the Hebei University of Science and Technology, China, in 2005, the M.S. degree in computer application from Yanshan University, China, in 2009, and the Ph.D. degree in control science and engineering from Tianjin University, China, in 2013. He is currently with the College of Electronic Information and Automation, Tianjin University of Science and Technology. His current interests include multi-phase flow parameter measurement, flow pattern identification, time series analysis, and machine learning.

Mr. Hongyi Yin
$\begin{aligned} & \text { He received the B.S. degree in communica- } \\ & \text { tion engineering from Qiqihar University, } \\ & \text { China, in 2017. He is currently pursuing } \\ & \text { the M.S. degree in control science and engi- } \\ & \text { neering with the Department of Electronic } \\ & \text { Information and Automation, Tianjin } \\ & \text { University of Science and Technology, } \\ & \text { China, under the supervision of Dr. M. Du. } \\ & \text { His current research interests include }\end{aligned}$
multi-phase flow parameter measurement, flow pattern identifi-
cation, and machine learning.

